\title{
How to excite a rogue wave
}

\author{
N. Akhmediev, ${ }^{1}$ J. M. Soto-Crespo, ${ }^{2}$ and A. Ankiewicz ${ }^{1}$ \\ ${ }^{1}$ Optical Sciences Group, Research School of Physical Sciences and Engineering, The Australian National University, Canberra, \\ Australian Capital Territory 0200, Australia \\ ${ }^{2}$ Instituto de Óptica, CSIC, Serrano 121, 28006 Madrid, Spain
}

(Received 30 July 2009; published 19 October 2009)

\begin{abstract}
We propose initial conditions that could facilitate the excitation of rogue waves. Understanding the initial conditions that foster rogue waves could be useful both in attempts to avoid them by seafarers and in generating highly energetic pulses in optical fibers.
\end{abstract}

DOI: 10.1103/PhysRevA.80.043818

PACS number(s): 42.81.Dp, 42.65.Sf, 47.20.Ky

\section{INTRODUCTION}

Rogue waves are one of those fascinating destructive phenomena in nature that have not been fully explained so far [1-3]. There is a variety of approaches to deal with rogue waves $[4,5]$. Oceanographers commonly agree that linear theories cannot provide explanations for their existence [6,7]. Only nonlinear theories can explain the dramatic concentration of energy into a single "wall of water" well above the average height of the surrounding waves $[3,8,9]$. Among nonlinear theories, the most fundamental is based on the nonlinear Schrödinger equation (NLSE) [6]. If the fundamental approach allows us to give a basic explanation, then it can be extended to more general ones which take into account the two-dimensional nature of the problem, effects of the bottom friction [10], etc. Thus, starting from the simplest model is essential for understanding the phenomenon.

Scientists also agree that the initial process that leads to the formation of a rogue wave is modulation instability $[8,11,12]$. In common understanding, small periodic perturbations lead to high wave periodic structures during evolution. Generally, perturbations do not necessarily have to be periodic. The appearance of single bumps is more likely when the initial conditions are random or have a specific form. One of the possible formation mechanisms for rogue waves is the creation of Akhmediev breathers (ABs) $[10,13-15]$ that appear due to modulation instability. Then, larger rogue waves can build up when two or more $A B s$ collide [16].

Rogue waves in the ocean are naturally born from random initial conditions. These conditions can later create a multiplicity of $\mathrm{ABs}$ appearing in all possible positions along the surface of the ocean. Double and triple collisions lead to the appearance of either high or giant rogue waves with amplitudes two or three times higher than the average wave crests in the surrounding area.

Rogue waves can also be observed in optics when propagating optical radiation in photonic crystal fibers $[17,18]$. Until now, only randomly created rogue waves have been observed experimentally [17]. The processes here are very similar to what happens in the ocean: we observe all random waves and then select only the highest peaks as prototypes of rogue waves.

Clearly, we cannot do much if we leave the creation of rogue waves to chance. On the other hand, preparing special initial conditions could be useful. Thus, the next question that we ask ourselves is the following: what are those specific initial conditions that lead directly to the appearance of rogue waves? Understanding this issue would be useful both in attempts to avoid rogue waves in the way of seafarers and in generating highly energetic pulses emerging from optical fibers.

Among previous approaches to this problem, we should mention the ideas of Pelinovsky and co-workers (see [19] and the book [20] for a review). Namely, starting the evolution from an initial sharp Gaussian or delta function leads to subsequent dispersion to smaller amplitude waves. Inverting the process in time would generate a high amplitude rogue wave from the initial conditions found in the direct process. However, having analytical expressions for the initial conditions and for the final results is more appealing from both mathematical and experimental points of view.

Understanding the nature of rogue waves can lead to a better way to suppress or generate them. In optics, we could then create them systematically and obtain high energy pulses each time when we want them. This work suggests a technique for creating rogue waves out of optical fiber devices.

As before [16,21], we deal here with the standard "selffocusing" NLSE. In dimensionless form, it is given by [22]

$$
i \frac{\partial \psi}{\partial x}+\frac{1}{2} \frac{\partial^{2} \psi}{\partial t^{2}}+|\psi|^{2} \psi=0,
$$

where $x$ is the propagation variable and $t$ is the transverse variable. The notation here for the independent variables $x$ and $t$ is reversed in comparison with the convention taken in the classical work by Zakharov and Shabat [23]. However, our convention is standard in the theory of ocean waves [9] and waves in optical fibers [24], so we use it throughout this paper. In each case, the function $\psi$ describes the envelope of the modulated waves, and its absolute value carries information about either wave elevation above the water surface or the intensity of an optical wave.

\section{DOUBLE AND TRIPLE COLLISIONS OF ABS}

$\mathrm{ABs}$ and their nonlinear superpositions can be constructed using a Darboux transformation scheme [16,25]. We have studied collisions of two ABs constructed this way in [16]. 
(a)

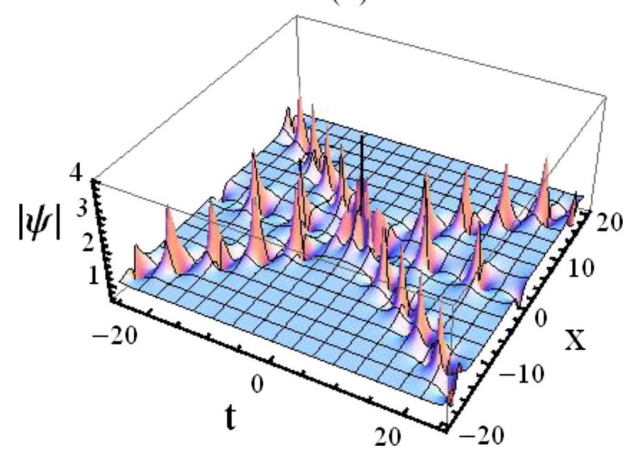

(b)

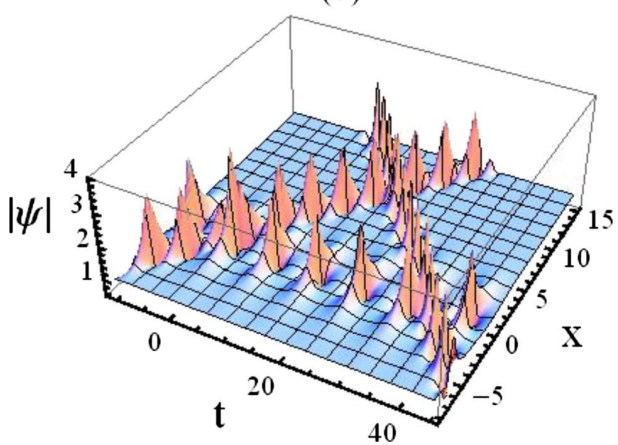

FIG. 1. (Color online) (a) Collision of three ABs with eigenvalues: $l_{1}=0.2+i 0.95, l_{2}=+i 0.92$, and $l_{3}=-0.2+i 0.95$. Coordinate and phase offsets are set to zero. All three ABs collide at one point with the resulting highest maximum amplitude being around 7 . (b) Collision of three ABs with eigenvalues: $l_{1}=0.5+i 0.95, l_{2}=+i 0.92$, and $l_{3}=-0.5+i 0.95$. Coordinate offsets: $t_{0}=12$ and $x_{0}=-12$. There are three points of collision with potential maximum amplitude around 5 in each case provided that the phase offsets at those points are 0 . This is not the case here.

Extending the scheme to cover triple collisions shows that the amplitude of rogue waves in this case can be much higher. A particular case is shown in Fig. 1(a). The parameters are chosen in such a way that all three ABs cross at a single point, thus leading to a local increase in the amplitude at this point to a value close to 7 (not seen in the vertical scale of the figure). This is a very special case, as generally the three ABs will intersect at three different points on propagation [see Fig. 1(b)]. Then the amplitude at each of these points will be much lower. This example shows that the collision of three $\mathrm{ABs}$ in a single point should be a rare event. If we want to realize such collision in practice, we need to create special initial conditions.

There is one more reason why high amplitudes are rare. In the case of Fig. 1(a), in order to have the maximum amplitude, the periodic structures in each $\mathrm{AB}$ have to be synchronized, i.e., all three $\mathrm{ABs}$ must provide constructive superposition of three $\mathrm{ABs}$ at the point of intersection. A simple triple collision does not ensure that the amplitude would be the highest. This reason further reduces the probability of appearance of rogue waves with the highest possible amplitude. The requirement of phase synchronization is valid for double collisions as well. Nevertheless the chances of double collision of ABs with the highest amplitude are still higher of course.

If we consider the wave disturbance (with amplitude around 5) at the intersection of two $\mathrm{ABs}$, each with $l_{i} \approx 1$, then we can refer to it as a strong rogue wave. It resembles a $j=2$ rational solution [21]. If the rogue wave is a wave disturbance produced by the simultaneous collision of three ABs, with an amplitude close to 7 (i.e., $j=3$ ), we can call it a superstrong rogue wave. Higher-order collisions are progressively less likely although not completely impossible.

With regard to ocean waves, even a single $\mathrm{AB}$ by itself is a rogue wave [14]. We showed [16] that a low-order rational solution of order $j$ has amplitude $2 j+1$. Furthermore, a single $\mathrm{AB}$ with eigenvalue $l_{r}+i l_{i}$ has infinitely many peaks in a line and each has amplitude $2 l_{i}+1$. The first-order rational solution can be viewed as the limit of the $\mathrm{AB}$ with $l_{r} \rightarrow 0$ and $l_{i} \rightarrow 1$, so it has a single maximum with amplitude 3 .

A single $A B$ in an optical fiber has previously been observed by Van Simaeys et al. [26]. In that case, single fre- quency modulation of $\mathrm{cw}$ radiation was enough to generate that effect. Hence, generating a single $\mathrm{AB}$ is relatively simple. To observe double and triple collisions requires much more complicated initial conditions. Indeed, generation of three ABs simultaneously in Figs. 1(a) and 1(b) requires special efforts. Although the solution can be expressed analytically, it is clear that, experimentally, creating these collisions would be hard to arrange. Thus, in this paper we give a simple analysis to show what kind of initial condition is needed to create strong and superstrong rogue waves in optical fibers.

\section{TWO TYPES OF ABS}

In contrast to ordinary solitons, $\mathrm{ABs}$ with nonzero velocities can be obtained in two different ways. First, we can start with the zero velocity one-parameter family of ABs $[27,28]$,

$$
\psi_{1}=\left[1-\frac{\kappa_{1}^{2} \cosh \delta_{1} x+2 i \kappa_{1} \nu_{1} \sinh \delta_{1} x}{2\left(\cosh \delta_{1} x-\nu_{1} \cos \kappa_{1} t\right)}\right] \exp [i(x+\pi)],
$$

where $\delta_{1}=\nu_{1} \kappa_{1}$ and $\kappa_{1}=2 \sqrt{1-\nu_{1}^{2}}$, which has purely imaginary eigenvalue $l_{1}=i \nu_{1}$ (real $\nu_{1}$ is the parameter of the family) and apply the Galilean transformation to it,

$$
\psi(t, x)=\psi_{1}(t-V x, x) \exp \left(i V t-\frac{i V^{2} x}{2}\right) .
$$

Clearly, the solution obtained this way will also be a solution of the NLSE. It is shown in Fig. 2(a). The main feature of this solution is that all maxima of the $\mathrm{AB}$ are located on the line $x=0$, as they were without the Galilean transformation. The only difference is that the ridges are now tilted in the $(x, t)$ plane. However, the plane wave background for this solution propagates in a direction that does not coincide with the $x$ axis. On the other hand, if we want to study collisions of several ABs, we have to use a common background for all of them. Thus, we cannot use a Galilean transformation to rotate $\mathrm{ABs}$, as is usually done with soliton solutions. The result will not be the same as when using complex eigenval- 
(a)

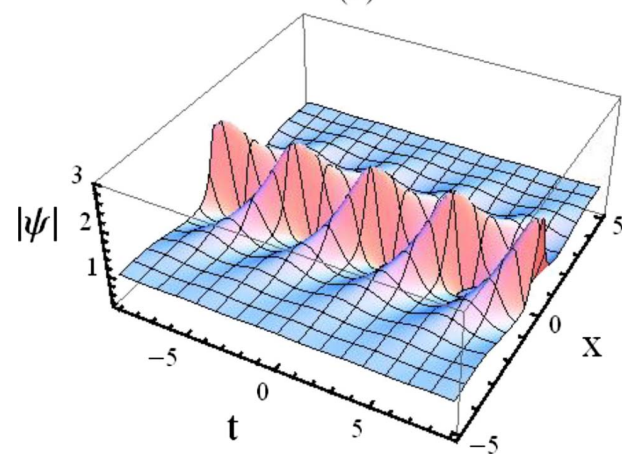

(b)

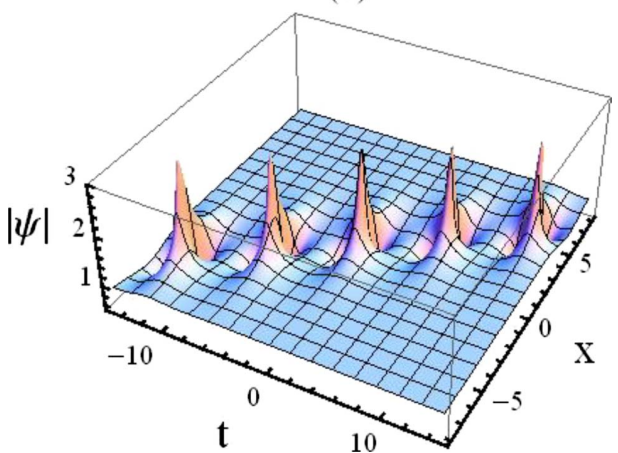

FIG. 2. (Color online) Two types of ABs with nonzero velocity. (a) Galilean transformation is applied to AB with zero velocity. Here $V=1$ and $\nu_{1}=0.7$. (b) Direct calculation of $\mathrm{AB}$ with an eigenvalue $l_{1}=-0.08+i 0.9$.

ues from the beginning. This essential difference between ABs and soliton solutions of the NLSE is crucial for the discussions that follow below.

If we use a complex eigenvalue from the beginning of the derivation of an $\mathrm{AB}$, we obtain a different $\mathrm{AB}$. (For details see Appendix A in [16].) This is shown in Fig. 2(b). Now, the line of maxima of the $\mathrm{ABs}$ is tilted with respect to the line $x=0$, but, in contrast to the previous case, the elongated ridge associated with each peak seems to be in line with the propagation direction. The background plane wave here is propagating along the $x$ axis, thus allowing us to construct a nonlinear superposition of different $\mathrm{ABs}$ on a common background.

Thus, in the second case, an $\mathrm{AB}$ is a periodic sequence of individual peaks located along a tilted line. We can define the velocity as the tangent of the angle between this line and the $t$ axis. Curiously, the velocity does not coincide with the real part of the eigenvalue, but it is related to it. We consider a general eigenvalue $l_{r}+i l_{i}$. As noted earlier, each peak in the line has amplitude $2 l_{i}+1$. We find that the resulting velocity of the breather, $v_{b}$, is given by

$$
v_{b}^{-1}=-\frac{l_{i}}{b}\left[\operatorname{sgn}(c) \sqrt{1+b^{2}}+1\right]-l_{r},
$$

where $c=1+l_{r}^{2}-l_{i}^{2}$ and

$$
b=\frac{2 l_{r} l_{i}}{c} .
$$

We plot this in Fig. 3. If $l_{r}$ and $l_{i}$ are both small, then

$$
v_{b} \approx-l_{r},
$$

so the velocity is approximately linear in $l_{r}$ in these cases, as shown by the blue curve. If $l_{i}=1$, then $b$ becomes $2 / l_{r}$ and the velocity simplifies to

$$
v_{b}=\frac{-2}{\left(3 l_{r}+\sqrt{l_{r}^{2}+4}\right)}
$$

(see red curve).

We can also determine the periods, $T_{x}, T_{t}$, in the $x$ and $t$ directions, respectively. We find

$$
T_{x}=\frac{2 \pi}{m^{2}}\left|-\frac{k_{i}}{l_{i}}\right|
$$

and

$$
T_{t}=\frac{2 \pi}{m^{2}}\left|k_{r}+\frac{k_{i} l_{r}}{l_{i}}\right| .
$$

Here $k_{r}=m \cos (A), \quad k_{i}=m \sin (A)$, where $A=[1-\operatorname{sgn}(c)] \frac{\pi}{4}$ $+\frac{1}{2} \arctan (b)$ and $m=2\left[c^{2}+\left(2 l_{r} l_{i}\right)^{2}\right]^{1 / 4}$. The ratio $T_{x} / T_{t}$ gives $\left|v_{b}\right|$.

If $l_{i}=1$ and $\left|l_{r}\right|$ is small, then

$$
T_{t} \approx T_{x} \approx \frac{\pi}{2 \sqrt{\left|l_{r}\right|}},
$$

so the periods approach infinity as $l_{r} \rightarrow 0$, and we arrive at a single peak at the origin. It is the $j=1$ rational solution [21].

\section{SINGLE AB INITIAL CONDITIONS}

The key question in studying ABs is how to excite them. Zero velocity $\mathrm{ABs}$ can naturally be excited by starting with modulation instability [27]. The latter develops when a $\mathrm{cw}$ is perturbed with a cosine periodic wave. In the spectral do-

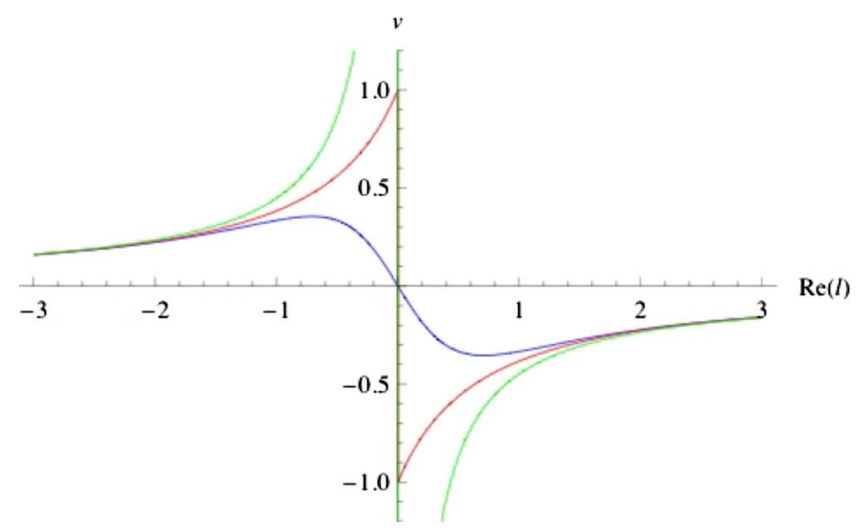

FIG. 3. (Color online) Velocity of a single $A B$ as a function of $l_{r}$. Here we have $l_{i}=0$ (blue curve passing through origin), $l_{i}= \pm 1$ (red curves giving velocity \pm 1 for small real part), and $l_{i}= \pm 2$ (green). 

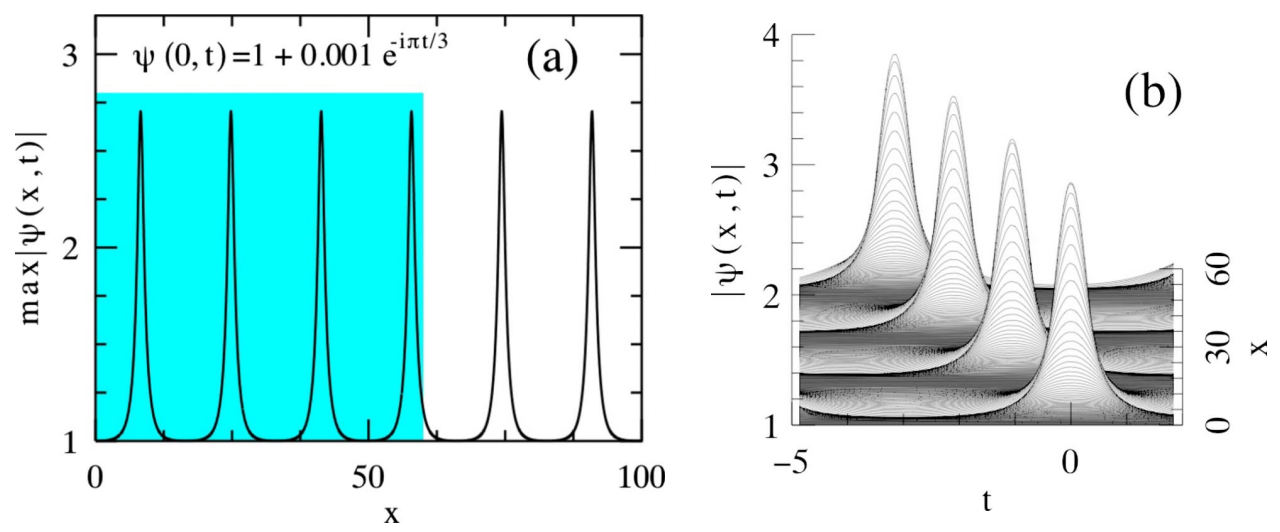

FIG. 4. (Color online) Excitation of a single AB with nonzero velocity with a single sideband in the spectrum. (a) Periodic evolution of the peak amplitude. (b) Evolution of the solution from $x=0$ to $x=60$ [blue shaded area in (a)].

main, a periodic perturbation corresponds to two symmetrically located sidebands around the central $\mathrm{cw}$ peak [27]. The strength of the sidebands defines the distance along the $x$ direction where the maxima of the $\mathrm{AB}$ appear. The modulation instability (MI) develops exponentially, so when the modulation is smaller, then the maxima of the ABs are further away in the $x$ direction.

Naturally, in order to create ABs that propagate at a nonzero angle to the line $t=0$, we should choose the two sidebands with a certain asymmetry. When one of the sidebands is stronger than the other one, the $\mathrm{AB}$ peaks will be located at an angle to the starting line. In particular, to have sidebands with considerable asymmetry, we can use a complex exponential function rather than a cosine one. The wave propagation evolution for the input $\psi(0, t)=1+0.001 \exp (-i \pi t / 3)$ is presented in Fig. 4. Indeed, this figure shows the creation of a single $A B$ which propagates at a certain angle with respect to the line $t=0$.

We can control the velocity of the $\mathrm{AB}$ by controlling the asymmetry of the two sidebands. Specifically, by choosing a superposition of two complex exponentials, viz., $\psi(0, t)=1$ $+C \exp (-i \omega t)+D \exp (i \omega t)$, as an input function, the velocity of the resulting breather can be selected by an appropriate choice of the small initial real amplitudes $C$ and $D$. The parameter $\omega$ is the frequency of the initial perturbation. It has to be inside the modulation instability gain curve for the instability to develop. Strictly speaking, these initial conditions create $\mathrm{ABs}$ to a certain degree of accuracy. The line of maxima of $\mathrm{ABs}$ with nonzero velocity always crosses the initial condition line, thus perturbing a simple exponential function. The problem is illustrated in Fig. 5. As we can see from this figure, the initial modulation has to have a complicated amplitude component. However, for our aims, this mismatch is not dramatically important, as it will only create some additional radiation waves.

In order to create two or more ABs, like those in Fig. 1, which eventually will collide, we can make a superposition of several exponential functions on top of a plane wave. We also have to take care of the phases of exponential functions, as the collision point has to coincide with the maxima of the two ABs. To be specific, we give a few examples below.

\section{INITIAL CONDITIONS FOR CREATING TWO COLLIDING ABS}

In this case, we need two pairs of sidebands so that we can excite two ABs. Thus, let us take as initial conditions the following:

$$
\psi(0, t)=1+A_{1} \exp (i \omega t)+A_{2} \exp \left(i \omega^{\prime} t\right)
$$

where the frequencies $|\omega|$ and $\left|\omega^{\prime}\right|$ are smaller than 2 to be inside the MI gain curve and $A_{1}$ and $A_{2}$ are two small real amplitudes to adjust so that we can control the initial ratio of the two perturbations and therefore the approximate distance at which the two ABs will reach their respective maxima. These conditions are highly asymmetric and they are expected to create two ABs, with different velocities colliding after a certain distance, $x_{0}$.

Figure 6 shows the peak amplitude versus the propagation distance for the initial condition [Eq. (6)] with $A_{1}=0.01, A_{2}$ $=0.001, \omega_{1}=\pi / 4$, and $\omega_{2}=\pi / 2$. We stress that we plot the maximum amplitude calculated for all values of $t$ here. Two sets of peaks of different amplitude can be observed, corresponding to the evolution of two different ABs. At some value of $x(x \approx 91.4)$ they collide and give rise to a high maximum which we define as a rogue wave. Having this example, we can, with some certainty, design the initial con-

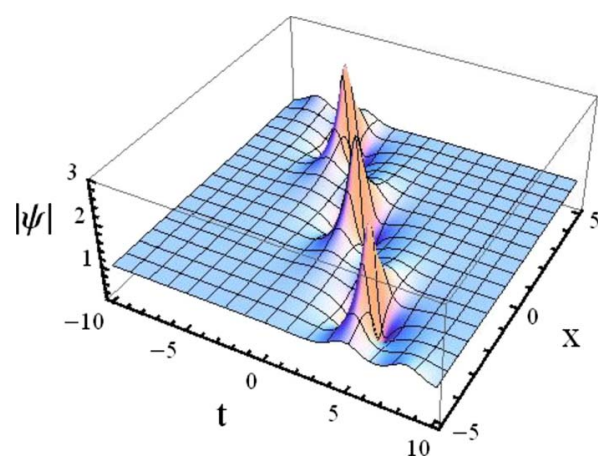

FIG. 5. (Color online) Single AB with eigenvalue $l=0.2+i$. The $\mathrm{AB}$ crosses the input line, giving a complicated wave envelope at $x=-5$. This example shows that the initial conditions are not as trivial as those considered in Fig. 4. 


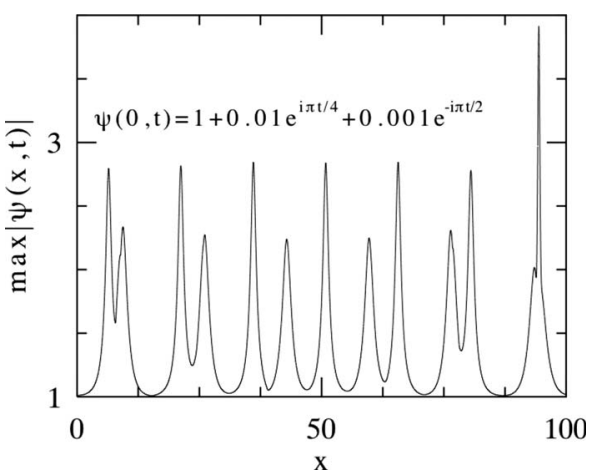

FIG. 6. Evolution of the peak amplitude when the initial cw has two harmonics of modulation. The high peak at the end of simulation is due to the in-phase collision of two ABs.

ditions that generate two colliding $\mathrm{ABs}$ with a high peak at the collision point. We can vary the small initial amplitudes and frequencies of the exponentials to generate their collision as the resulting rogue wave after the shortest possible distance, $x_{0}$.

Figure 7(a) shows the evolution of the peak amplitude for three different values of $A_{1}$ and $A_{2}$ and fixed values of $\omega_{1}$ $=2 \pi / 13.2$ and $\omega_{2}=5 \pi / 13.2$. The inset shows the position of these two frequencies inside the MI gain curve. For the case depicted by the solid red line, a high maximum is obtained as a result of a fully synchronized collision of the two generated ABs with the amplitude of the rogue wave being the highest possible for these frequencies. A contour plot of the evolution of this case from $x=5$ to $x=15$ is shown in (b), where the location of the maximum is encircled with a dashed line. Due to the spatial periodicity of the two ABs, they can intersect at different points of their local maxima. Thus, the combined evolution is not necessarily periodic and does not necessarily produce a high peak. As mentioned above, the highest maximum can only be obtained when both ABs collide in such a way that their local maxima coincide at the same point of the plane $(x, t)$. We describe this as an "inphase" or "synchronized" collision. Once we fix the amplitudes in the exponential functions, the probability of a high peak can be estimated using the principles of constructive interference. For example, for the peak to reach at least $90 \%$ of the maximum value, the probability is 0.15 . In the two other cases shown in Fig. 7 by green dashed and blue dotted curves, either $A_{1}$ or $A_{2}$ is set to zero and only one $\mathrm{AB}$ is excited. Thus the curves do not reach such high maxima as before.

\section{COLLISION OF MORE THAN TWO ABS}

Figure 1 shows clearly that increasing the number of $\mathrm{ABs}$ increases the chances of collisions with even higher amplitudes. The chances of triple collision are lower, of course, but not zero. A complete theory of initial conditions for multiple modulation still has to be developed. We give here (Fig. 8) only one example which involves six ABs generated by multiple initial modulation. The lowest frequency of this initial modulation is so small that even its sixth harmonic is located inside the MI gain curve. The lowest frequency (red solid vertical line) and its harmonics (blue dotted vertical lines) located within the modulation instability gain band are shown in the inset of Fig. 8. In our simulations, we only had the lowest harmonic in the initial conditions. All higher harmonics were excited due to four-wave mixing process. The only parameter that we varied was the amplitude of the lowest harmonic. The amplitudes of the higher harmonics were dependent on the amplitude of the lowest one. Thus, all amplitudes can be considered as self-organized and controlled by the lowest order one.

Without careful adjustment of the initial amplitudes and phases of these six harmonics, the synchronization of the collision of the ABs is left to chance. Using this chance, we can monitor the situation looking at the amplitudes of the high peaks. Any amplitude that is higher than 3 would be the result of a collision of two ABs, while any peak that is higher than 5 clearly involves the collision of three ABs. The curve shown in Fig. 8 clearly demonstrates that the amplitude of the rogue wave that is created is around 6, thus confirming the fact that it is the result of triple $\mathrm{AB}$ collision.

Generating rogue waves in numerical simulations by chance is not quite a satisfactory exercise. After all, we can
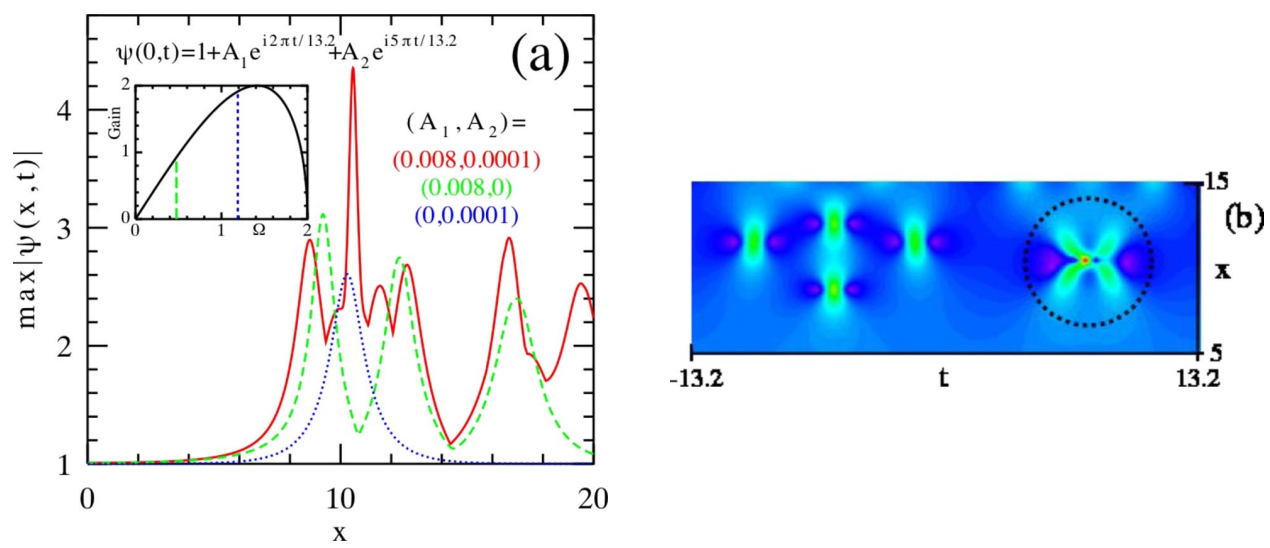

FIG. 7. (Color online) (a) Evolution of the maximum of the wave envelope when the initial cw is perturbed by two harmonics. The initial condition is written at the top of part of this figure. The inset shows the location of the two frequencies within the MI gain curve. The three curves are calculated for the set of amplitudes $A_{i}$ written inside the plot. Only one of these sets produces a rogue wave with a high peak (red solid curve). Its evolution in the $(x, t)$ plane is shown in (b). 

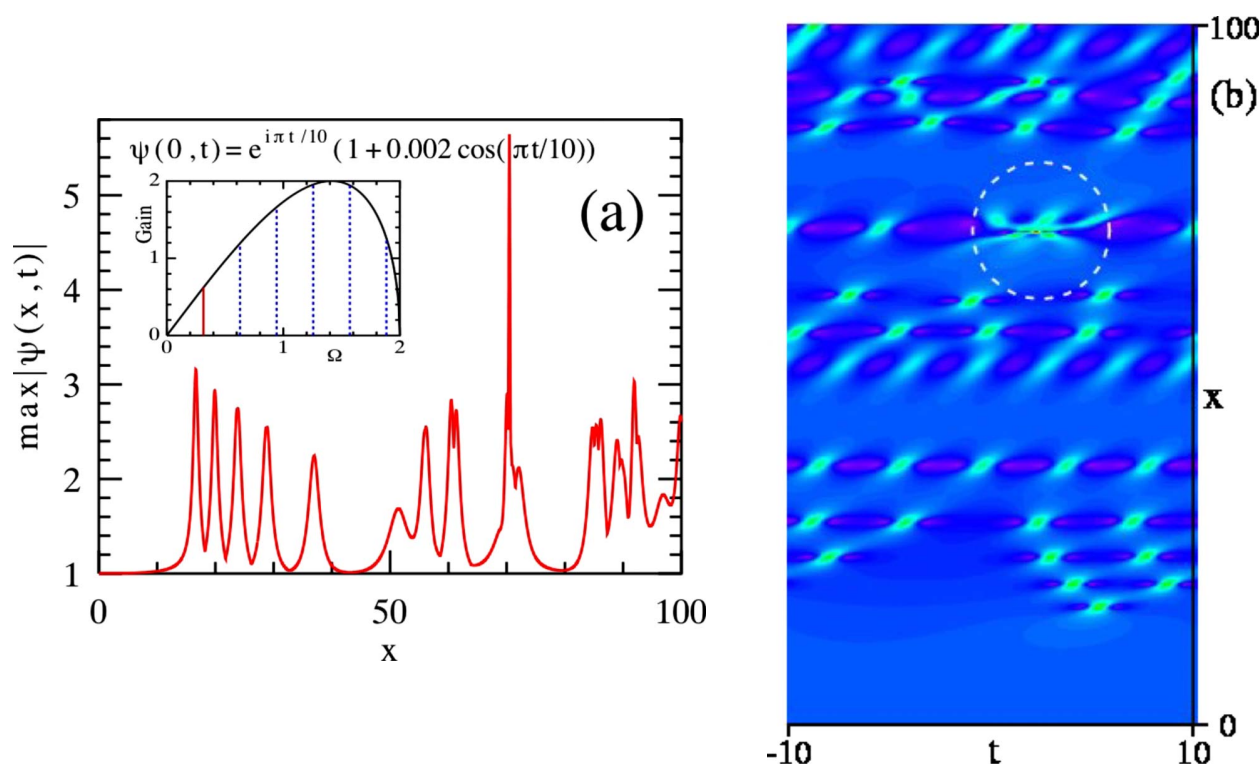

FIG. 8. (Color online) (a) Maxima of the wave envelope vs $x$ for the initial condition written on top of the figure. The location of the frequency of the initial perturbation and its harmonics within the MI gain band is shown in the inset. (b) Contour plot of the wave amplitudes in the $(x, t)$ plane. The point in the middle of the dashed circle corresponds to the high amplitude rogue wave with the highest amplitude in (a).

present exact solutions in analytical form and show them accurately, as in Fig. 1. However, creating initial conditions to generate those exact solutions is another matter. The unavoidable presence of radiation waves means that the process of generating $\mathrm{ABs}$ with nonzero velocities with simplified initial conditions is difficult. While we can have higher accuracy in the case of two ABs, the situation is hopeless when we deal with more than three ABs. In addition, it is very unlikely that exact initial conditions can be created in reality. In physics and in optics in particular, we deal with spectral harmonics of specific inputs and their amplitudes. Dealing with initial conditions at that simplified level may be sufficient to initiate experimental activity in the exciting area of generating rogue waves.

The stability of rogue waves is a natural question that is always posed when new nonlinear phenomena are considered. This question has many aspects. One aspect is the sensitivity of the peak amplitudes to small deviations of the parameters involved in this problem. Clearly, this problem is complicated and can only be answered on a step-by-step basis, considering each new perturbation separately. These studies are cumbersome and would require separate publications. The influence of third-order dispersion, selfsteepening, and a term related to the Raman effect has been considered recently in [29] with a positive answer: yes, there is stability. Other types of perturbations still need to be studied.

Finally, we should answer the following question: "are in-phase AB collisions structurally stable?" Namely, can any noise or small amplitude radiation waves destroy the high amplitude peaks found in this work? When we restrict ourselves to the integrable model such as NLSE in our case, the answer is yes, they are stable. According to the inverse scattering technique, for the NLSE, a general solution can be considered as a "nonlinear superposition" of elementary modes. The latter are ABs in our case. Hence, any additives in the form of small amplitude radiation may modify the peak profiles but cannot eliminate them. Although those peaks may seem to appear from nowhere, there is a high degree of inevitability in their appearance once the initial conditions are created.

\section{CONCLUSIONS}

In conclusion, we propose initial conditions that lead to the generation of rogue waves in optical fibers. For ocean waves, the theory cannot be applied directly, as the dynamics in two dimensions is more complicated. On the other hand, modeling of waves in long narrow water tanks could confirm the basic features of our theory. Subsequent expansion of these ideas to the case of the Dysthe equations [30] would also be a useful application of our theory. In optics, the knowledge of initial conditions will help to generate rogue waves on purpose in order to produce high energy light pulses. We hope that this work will initiate experimental efforts in these directions.

\section{ACKNOWLEDGMENTS}

N.A. and A.A. gratefully acknowledge the support of the Australian Research Council (Discovery Project No. DP0985394). J.M.S.-C. acknowledges support from the Spanish Ministerio de Ciencia e Innovación under Contracts No. FIS2006-03376 and No. FIS2009-09895. 
[1] P. Müller, Ch. Garrett, and A. Osborne, Oceanogr. 18, 66 (2005).

[2] C. Kharif and E. Pelinovsky, Eur. J. Mech. B/Fluids 22, 603 (2003).

[3] A. A. Kurkin and E. N. Pelinovsky, Killer-Waves: Facts, Theory and Modeling (Nizhny Novgorod University Press, Nizhny Novgorod, Russia, 2004) (in Russian).

[4] P. A. E. M. Janssen, J. Phys. Oceanogr. 33, 863 (2003).

[5] M. Onorato, A. R. Osborne, M. Serio, and S. Bertone, Phys. Rev. Lett. 86, 5831 (2001).

[6] A. R. Osborne, Nonlinear Ocean Waves (Academic Press, New York, 2009).

[7] P. K. Shukla, I. Kourakis, B. Eliasson, M. Marklund, and L. Stenflo, Phys. Rev. Lett. 97, 094501 (2006).

[8] D. H. Peregrine, J. Aust. Math. Soc. Ser. B, Appl. Math. 25, 16 (1983).

[9] A. R. Osborne, Mar. Struct. 14, 275 (2001).

[10] V. V. Voronovich, V. I. Shrira, and G. Thomas, J. Fluid Mech. 604, 263 (2008).

[11] T. B. Benjamin and J. E. Feir, J. Fluid Mech. 27, 417 (1967).

[12] V. I. Bespalov and V. I. Talanov, Zh. Eksp. Teor. Fiz. Pis'ma Red. 3, 471 (1966) [JETP Lett. 3, 307 (1966)].

[13] V. I. Shrira and V. V. Georgaev (private communication).

[14] K. B. Dysthe and K. Trulsen, Phys. Scr. T82, 48 (1999).

[15] I. Ten and H. Tomita, Reports of RIAM Symposium No. 17SP1-2, Chikushi Campus, Kyushu University, Kasuga, Fukuoka, Japan (Kyushu University Press, Kyushu, 2006), Article No. 04.

[16] N. Akhmediev, J. M. Soto-Crespo, and A. Ankiewicz, Phys. Lett. A 373, 2137 (2009).
[17] D. R. Solli, C. Ropers, P. Koonath, and B. Jalali, Nature (London) 450, 1054 (2007).

[18] D.-Il. Yeom and B. Eggleton, Nature (London) 450, 953 (2007).

[19] E. Pelinovsky, T. Talipova, and C. Kharif, Physica D 147, 83 (2000).

[20] C. Kharif, E. Pelinovsky, and A. Slunyaev, Rogue Waves in the Ocean, Observations, Theories and Modeling, Advances in Geophysical and Environmental Mechanics and Mathematics Series (Springer, Heidelberg, 2009), Vol. XIV.

[21] N. Akhmediev, A. Ankiewicz, and M. Taki, Phys. Lett. A 373, 675 (2009).

[22] N. Akhmediev and A. Ankiewicz, Solitons, Nonlinear Pulses and Beams (Chapman and Hall, London, 1997).

[23] V. E. Zakharov and A. B. Shabat, Zh. Eksp. Teor. Fiz. 61, 118 (1971) [Sov. Phys. JETP 34, 62 (1972)].

[24] A. Hasegawa and F. Tappert, Appl. Phys. Lett. 23, 142 (1973).

[25] N. Akhmediev, V. I. Korneev, and N. V. Mitskevich, Zh. Eksp. Teor. Fiz. 94, 159 (1988) [Sov. Phys. JETP 67, 89 (1988)].

[26] G. Van Simaeys, Ph. Emplit, and M. Haelterman, Phys. Rev. Lett. 87, 033902 (2001).

[27] N. Akhmediev and V. I. Korneev, Teor. Mat. Fiz. 69, 189 (1986) [Theor. Math. Phys. (USSR) 69, 1089 (1986)].

[28] N. Akhmediev, V. M. Eleonskii, and N. E. Kulagin, Teor. Mat. Fiz. 72, 183 (1987) [Theor. Math. Phys. (USSR) 72, 809 (1987)].

[29] A. Ankiewicz, N. Devine, and N. Akhmediev, Phys. Lett. A 373, 3997 (2009).

[30] K. B. Dysthe, Proc. R. Soc. London, Ser. A 369, 105 (1979). 